\title{
Efecto del veneno de Bothrops diporus sobre la expresión de laminina 5 en biopsias de pie equino
}

\author{
EFFECT OF THE VENOM OF Bothrops diporuS ON THE EXPRESSION OF LAMININE 5 IN EQUINE \\ FOOT BIOPSIES \\ Pamela Teibler $^{1}$, María Aguirre², Juan Todaro²; Tania Stoyanoff ${ }^{2}$, Sandra Núñez ${ }^{1}$, \\ Silvana Maruñak ${ }^{1}$
}

\section{ReSUMien}

El objetivo del presente trabajo fue estudiar la expresión de laminina 5 en cortes de biopsias de pie equino precultivados con veneno de serpiente Bothrops diporus por histopatología e inmunohistoquímica contra controles no tratados. Las membranas basales de las láminas de las biopsias, así como las de las arteriolas y venas interlamelares evidenciaron intensa inmunomarcación de laminina 5 (L-5) en los cultivos controles; sin embargo, fue significativamente menor en las biopsias precultivadas con el veneno de $B$. diporus concomitantemente con la disrupción de la histoarquitectura típica de las laminillas. Estos resultados describen por primera vez la normo-localización de L-5 en biopsias de pie equino y el efecto de las metaloproteinasas, componentes de los venenos de $B$. diporus, tanto en la integridad lamelar como en los componentes de la vascularización.

Palabras clave: laminina 5; expresión; biopsia; veneno; cultivo; pie equino

\section{AbSTRACT}

The aim of the present study was to study the expression of laminin 5 in equine foot biopsies precultured with venom of Bothrops diporus by histopathology and immunohistochemistry against untreated controls. The basal membranes of the laminae of the biopsies, as well as those of the interlamellar arterioles and veins, showed intense immunostaining of laminin 5 (L-5) in the control cultures; however, it was significantly lower in pre-cultured biopsies with the $B$. diporus venom concomitantly with the disruption

\footnotetext{
${ }^{1}$ Facultad de Ciencias Veterinarias, Universidad Nacional del Nordeste - UNNE, Argentina

${ }^{2}$ Facultad de Medicina, Universidad Nacional del Nordeste - UNNE, Argentina

${ }^{3}$ E-mail: pteibler@vet.unne.edu.ar
}

Recibido: 9 de noviembre de 2017

Aceptado para publicación: 4 de abril de 2018 
of the typical laminar plate histoarchitecture. These results describe for the first time the normo-localization of L-5 in equine foot biopsies and the effect of the metalloproteinases, components of $B$. diporus venoms, both in the lamellar integrity and in the components of the vascularization.

Key words: laminin 5; expression; biopsy; venom; culture; equine foot

\section{INTRODUCCIÓN}

La unión dermo-epidérmica del pie equino es una estructura que recubre a la tercera falange y se caracteriza por ser celular y avascular (epidermis) que depende del intercambio gaseoso de sus células basales y de la irrigación proporcionada por el tejido dérmico para su adecuada nutrición (Pollitt 1998a,b). Esto lo constituye en una región anatómica muy vascular y, dada su accesibilidad, se torna favorable para la obtención de biopsias destinadas al estudio de posibles efectos adversos de sustancias tóxicas sobre la estructura vascular.

Diferentes etiologías con acción sistémica en el paciente pueden alterar la red vascular y otros componentes de la unión dermo-epidérmica del pie equino causando laminitis (McGowan 2008; Bailey et al., 2009), patología que se caracteriza por intenso dolor y desprendimiento de láminas con las consecuencias que ello provoca. Así, por ejemplo, las intoxicaciones por veneno de serpientes inducen una serie de efectos locales y sistémicos, incluyendo laminitis (Dickinson et al., 1996; Acosta et al., 2006). En mordeduras de ofidios se observa que, si bien las alteraciones sistémicas se controlan luego de la administración del suero antiofídico, ocurren lesiones permanentes en el casco del miembro afectado (Dickinson et al., 1996; Acosta y Brem, 1997).

Las serpientes del género Bothrops, pertenecen a la familia Viperidae. Sus venenos inducen daños locales como hemorragia, mionecrosis y edema (Gay et al., 2005), su- mado a efectos sistémicos tales como desórdenes dela coagulación (Bogado et al., 2013), shock cardiovascular y falla renal aguda. Estos efectos son inducidos por una variedad de componentes, tales como fosfolipasas $\mathrm{A}_{2}$ (Garcia Denegri, 2011), enzimas tipo trombina (Vieira et al., 2004) y metaloproteinasas, entre otras (Wang y Huang 2002). Es probable que las hemorraginas que son metaloproteinasas (Gutiérrez y Rucavado 2000; Gay et al., 2009; Oliveira et al., 2010) alteren la circulación del pie y provoquen alteraciones de las láminas dérmicas (Moore et al., 2004; Teibler et al., 2017). Otras sustancias del veneno inducen a inflamación, como las PLA $_{2}$ (Garcia Denegri, 2011) a lo que se le suma la reacción propia del animal, con liberación de mediadores tales como histamina, serotonina, prostaglandinas y otros (Texeira et al., 2009).

En la estructura del pie se encuentra la membrana basal (MB), estructura compleja de proteínas que constituye una importante barrera para el pasaje del material entre el epitelio y los compartimientos del tejido conectivo (Pestronk et al., 1982). La MB es responsable de la unión de las láminas dérmica, epidérmica y, consecuentemente, de soportar todo el peso corporal. Está compuesta mayormente por colágeno, laminina y proteoglicanos (LeBleu et al., 2007) y formada por tres capas: la lámina densa, la lámina lúcida, próxima a la membrana de las células y la lámina interna reticularis, cuya periferia se confunde con el colágeno de células inmersas en la matriz extracelular (MEC) vecina, secretado tanto por las células endoteliales como por las células estromales vecinas (Katwa et al., 1999). La 
lámina densa está constituida por colágeno tipo IV, cuya estructura a diferencia de los otros colágenos, no es fibrilar sino multilaminada y su estructura de triple hélice le da a la MB una plasticidad especial.

Las lamininas son componentes de la MEC que contribuyen a la arquitectura de las membranas basales y median la adhesión celular, el crecimiento, la migración y la diferenciación (Yurchenco y Schittny, 1990). La localización de variantes específicas de tejidos sugiere que cada isoforma de laminina cumple funciones particulares (Engvall, 1993). La L-5 se encuentra en la zona de la membrana basal de la piel, la tráquea, el tracto alimentario y otros epitelios escamosos especializados en funciones secretoras o protectoras. Esta proteína heterotrimérica es sintetizada y secretada por las células epiteliales basales (Aberdam et al., 1994). En la piel se encuentra con los filamentos de anclaje de la lámina lúcida, estructuras filamentosas que unen las hemidesmosomas a la lámina densa de la unión dermo-epidérmica (Rouselle et al., 1991; Verrando et al., 1993).

Como indica Pollitt (1996), las lesiones en la laminitis se caracterizan por la separación y desintegración de la membrana basal laminar de las células epidermales basales, resultando en una alteración de la anatomía de la pared interna del casco, presentándose una disociación de las láminas dérmicas y epidérmicas. En casos severos, el proceso puede causar la separación de la falange distal del casco. Diversos estudios señalan que la degradación de la MB es común en la mayoría de los tejidos epiteliales durante la laminitis equina, sugierendo el rol de factores dañinos sistémicos (Visser y Pollitt, 2011), donde la desintegración de las láminas está mediada por la liberación descontrolada y excesiva de las MMP activas (Pollitt, 1996).

Por otra parte, la acción de las MMP exógenas puede presentarse durante las intoxicaciones con venenos de ciertas especies ofídicas. Así, se reporta la existencia de MMP como componentes en el veneno de
B. diporus que podrían repercutir en alteraciones sistémicas, de la vascularización y en la estructura del tejido muscular (Teibler et al., 2017). Sin embargo, poco se sabe de la localización fisiológica de la L-5 en biopsias de equinos y del efecto de este veneno, rico en MMP, sobre la estructura de las láminas de cascos y de los componentes vasculares anexos. Por lo tanto, el objetivo del presente trabajo fue analizar los efectos que causa el veneno entero de $B$. diporus sobre el pie equino a partir de biopsias obtenidas de la zona de muralla.

\section{Materiales y MéTodos}

\section{Obtención y Conservación del Veneno}

Se obtuvo un pool de veneno de especímenes adultos de Bothrops diporus capturados en el nordeste argentino y mantenidos en el serpentario de la ciudad de Corrientes, Argentina. El procedimiento de extracción fue realizado por personal especializado siguiendo las normativas bioéticas internacionales. El veneno fue desecado a temperatura ambiente y mantenido a $-20^{\circ} \mathrm{C}$. Fue disuelto al momento de su uso con una solución salina de buffer fosfato $\mathrm{pH} 7.2$.

\section{Toma de Biopsias}

La toma de muestras se realizó por biopsias, a partir de la cara dorsal de la tercera falange de cinco equinos de raza indefinida, de 3 a 15 años de edad, en el quirófano del Hospital de Clínicas de la Facultad de Ciencias Veterinarias de la Universidad Nacional del Nordeste (UNNE), previo lavado intenso de los miembros. El procedimiento fue realizado por veterinarios siguiendo las normativas bioéticas internacionales y el protocolo aprobado por el Comité de Bioética de la institución.

Los animales fueron tranquilizados con maleato de acepromacina $1 \%$ a dosis de 0.05 $\mathrm{mg} / \mathrm{kg}$. Luego se realizó el adelgazamiento 
del casco a nivel de la cara dorsal de la tercera falange utilizando una escofina. Se procedió al bloqueo abaxial mediante anestesia perineural con lidocaína al 2\% a razón de 3 $\mathrm{ml}$. Posteriormente y después de frotar la pared del casco con solución limpiadora de povidona yodada, se procedió a aplicar la técnica descripta por Ribeiro et al. (2017) modificada. Para esto, se definió un área de $4 \mathrm{x} 1$ $\mathrm{cm}$ en la porción más dorsal de la cápsula del casco a una distancia de $3 \mathrm{~cm}$ distal a la banda coronaria. El tejido queratinizado se adelgazó hasta el punto en que el tejido de la pared del casco dorsal se volvería flexible cuando se presionara con el fórceps. A partir de ese punto, se procedió a la toma de muestra, se incidió el tejido laminar dentro de los límites internos del área adelgazada usando un bisturí (hoja $\mathrm{N} .{ }^{\circ} 24$ ) y se practicó un vendaje compresivo, previa colocación de torunda de gasa estéril embebida con povidona-iodo $3 \%$.

El tratamiento posquirúrgico consistió en el cambio de vendajes cada $12 \mathrm{~h}$ y antibioticoterapia con penicilinaestreptomicina $(22.000 \mathrm{UI} / \mathrm{kg})$ combinada con analgésicos (fenilbutazona a dosis de $2.2 \mathrm{mg}$ / $\mathrm{kg}$ cada $24 \mathrm{~h}$ ) durante un periodo de seis días.

\section{Procesamiento de las Muestras}

Las muestras fueron colectadas en recipientes estériles e inmediatamente reducidas a fragmentos más pequeños de aproximadamente $0.5 \times 0.1 \mathrm{~cm}$, incluyendo en el corte al menos cuatro (4) láminas primarias. Posteriormente se lavaron con solución fisiológica mediante pasajes sucesivos en cajas de Petri estériles, bajo flujo laminar, totalizando cinco pasajes o lavados (Pollitt, 1996). Las muestras fueron colocadas en placas de cultivo celular Cellstar ${ }^{\circledR}$ de 12 pozos, conteniendo medio de cultivo DMEM (Dulbecco's Modified Eagle's Medium, Sigma Aldrich).

Los cultivos de biopsias fueron adicionados con $50 \mu \mathrm{g}$ por pozo de veneno entero de $B$. diporus. Como control se cultivaron placas con biopsias y medio de cultivo. Las incubaciones se realizaron en estufa gaseada humidificada $\left(37^{\circ} \mathrm{C}, 5 \%\right.$ de dióxido de carbono y $95 \%$ de humedad) durante $48 \mathrm{~h}$.

\section{Histopatología}

Las muestras, al término de la incubación, fueron fijadas en formaldehído bufferado al $10 \%(\mathrm{pH} 7)$ por $24 \mathrm{~h}$ y procesadas con las técnicas histopatológicas clásicas y teñidas con hematoxilina eosina (H-E).

\section{Inmunohistoquímica}

Cortes de biopsias de pie equinos precultivados con veneno de serpiente $B$. diporus (4 $\mu \mathrm{m}$ de espesor) fueron desparafinados según procedimiento estándar y sometidos a desenmascaramiento antigénico con tripsina al $0.1 \%$ en cámara húmeda a $37^{\circ} \mathrm{C}$ durante $15 \mathrm{~min}$. Se realizó el bloqueo de las peroxidasas endógenas con $\mathrm{H}_{2} \mathrm{O}_{2}$ en metanol durante $20 \mathrm{~min}$ con agitación orbital. Las biopsias se incubaron con una dilución 1:50 del anticuerpo policlonal de conejo antilaminina 5 (Abcam, ab 14509, Canadá) toda la noche a $4{ }^{\circ} \mathrm{C}$. En los controles negativos se reemplazó el anticuerpo primario por PBS.

Luego de la incubación, los cortes se lavaron con PBS y se trabajó con el kit DAKO LSAB+/HRP kit (DakoCytomation), conteniendo el anticuerpo secundario biotinilado y estreptavidina. Tras dos lavados con PBS se utilizó el cromógeno DAB (DAKO kit) para revelación. Las muestras se contra-tiñeron con hematoxilina y se evaluó la inmunomarcación según escala semicuantitativa contra el control negativo: $(0)$ ausencia de expresión, $(+)$ expresión débil, $(++)$ expresión moderada. Se tomó como control positivo la inmunomarcación de laminina 5 de piel de ratón (zona axilar). Todas las muestras fueron examinadas a doble ciego por distintos observadores, registrándose microfotografías con Cámara Olympus Coolpix (magnificación: x100, x 400). 


\section{Resultados}

\section{Histopatología}

El pie equino está formado por varias porciones anatómicas, donde se destaca la unión dermo-epidérmica de la cara dorsal del pie, por ser la región blanco en la laminitis equina. En los cortes histológicos de las muestras control se observó a la microscopía óptica que el estrato laminar epidérmico conservó su estructura normal, donde las láminas primarias se inter-digitaban con el tejido dérmico. Las láminas epidérmicas secundarias, que se originan en las láminas primarias, mantuvieron un patrón de distribución perpendicular con proyecciones de puntas redondeadas. Los núcleos de las células germinales se encontraban dispuestos en forma ordenada y oblicua al borde externo de la membrana basal y presentaban morfología ovalada.

La dermis conservó la estructura normal de los vasos sanguíneos, terminales nerviosas, y abundante cantidad de fibras colágenas. Entre la unión del tejido dérmico y epidérmico se observó que la membrana basal no presentó discontinuidad y se hallaba contorneando las láminas dérmicas secundarias.

En las biopsias de tejido dermo-epidérmico de pie incubado con veneno de $B$. diporus se observaron alteraciones en la arquitectura de las láminas epidérmicas secundarias. Adicionalmente, se evidenció adelgazamiento y ondulaciones de las láminas epidérmicas secundarias (LES). Los núcleos de las células basales se encontraban redondeados y eventualmente localizados en el polo basal de las células. Las alteraciones inducidas por el veneno de $B$. diporus también afectaron la MB, la cual presentaba zonas de separación con respecto a la estructura laminar epidérmica; inclusive con pérdida de unión con las células basales. Asimismo, algunas células basales epidérmicas evidencia- ron nucleólisis. El desprendimiento de la MB abarcó desde la cara epidérmica y las capas de MB laminares libres permanecieron unidas al tejido conjuntivo (Figura 1).

\section{Inmunohistoquímica}

La expresión de L-5 en los cultivos de biopsias del grupo control las láminas mostró intensa inmunomarcación en la membrana de los endotelios de arteriolas y venas interlamelares. Asimismo, la inmunoreactividad fue claramente notable en toda la extensión de la membrana basal a nivel de la unión dermo-epidérmica de las biopsias normales no tratados.

La inmunomarcación fue significativamente menor en las biopsias precultivadas con veneno de $B$. diporus. La laminitis experimental causó la disminución de la inmunomarcación de la L-5 y pérdida del patrón normal de localización a nivel de la unión dermo-epidérmica (Figura $2 \mathrm{~A}, \mathrm{~B}$ ). La pérdida de L-5 conlleva a la falla de los anclajes de los filamentos proteicos que conectan la epidermis a la dermis. Por lo tanto, sin un complemento completo de filamentos anclados, se produce la separación en la unión dermo-epidérmica lamelar.

A nivel de vasos sanguíneos se observó disminución de la inmunoreactividad de la L5 , evidenciando que las MMP del veneno afectan no solo la estructura de las laminillas, sino también la estructura de las membranas basales de arteriolas y vénulas interlamelares (Figura $2 \mathrm{C}, \mathrm{D}$ ).

Este trabajo reporta por primera vez el doble efecto deletéreo de las MMP del veneno de $B$. diporus sobre las estructuras componentes de las láminas y laminillas de los cascos equinos y la alteración de la microvasculatura de la lámina que conduce a un microambiente hipóxico y proinflamatorio que afecta la correcta funcionalidad de la unión dermo-epidérmica. 


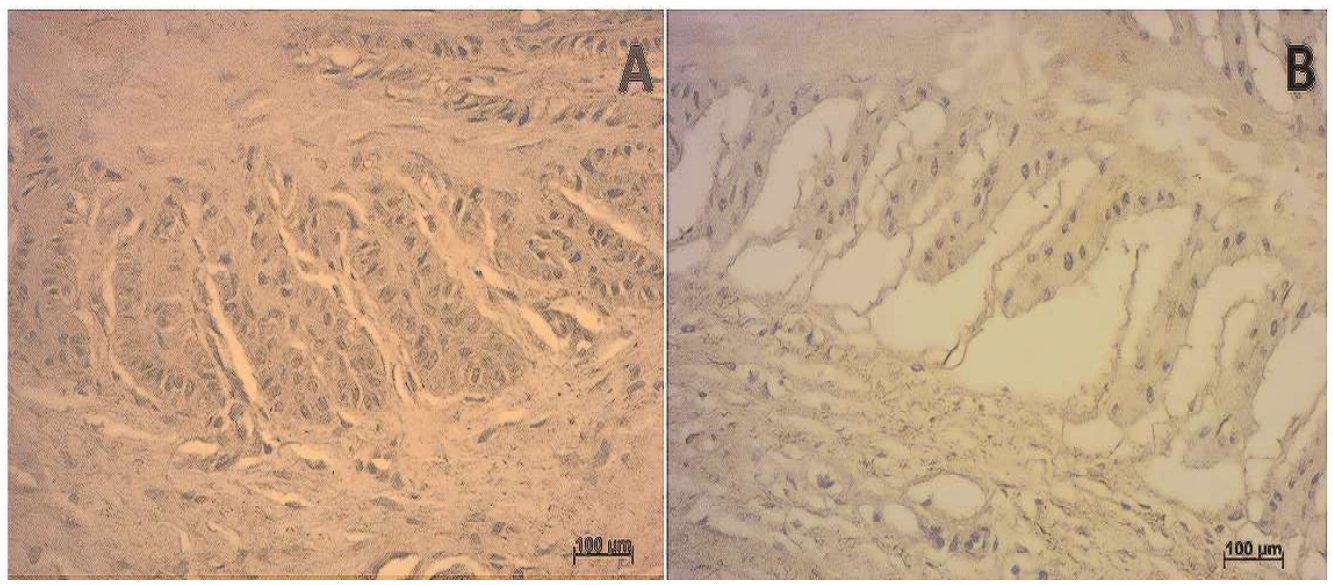

Figura 1. Evaluación histopatológica de biopsias de pie equino. A: Biopsia control. Se observa el tejido dermo-epidérmico normal con láminas epidérmicas secundarias (LES) unidas a las correspondientes láminas dérmicas secundarias (LDS). B: Biopsia de pie equino precultivado con veneno de Bothrops diporus. Se observa pérdida de la unión dermo-epidérmica y profunda alteración lamelar. Los espacios blanquecinos correspondientes a espacios de separación de LES de las LDS. Coloración H/E. Las barras indican $100 \mu \mathrm{m}$

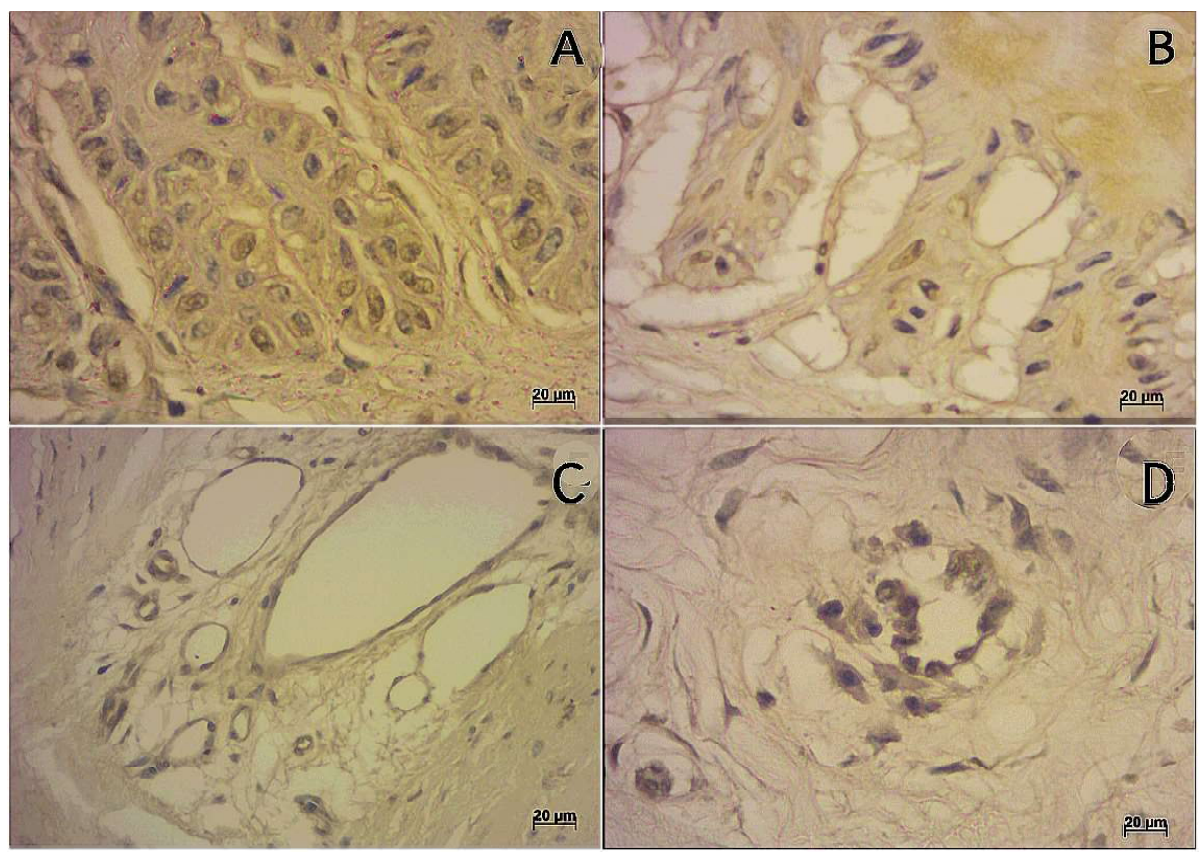

Figura 2. Inmunohistoquímica de laminina 5.A: Biopsia control con expresión fisiológica de laminina 5. La proteína de anclaje se encuentra uniformemente distribuida en las membranas basales a lo largo de la unión dermo-epidérmica. B: Biopsia de pie equino precultivado con veneno de Bothrops diporus. La expresión de laminina 5 es significativamente menor y se acompañan con pérdida de la integridad lamelar. Contra coloración nuclear con hematoxilina. Las barras indican $20 \mu \mathrm{m}$. C: Biopsia control con expresión fisiológica de laminina 5 (evaluación zona vascular). Se observa expresión constitutiva en membranas de vénulas y arteriolas. D: Biopsia de pie equino precultivado con veneno de $B$. diporus. La expresión de laminina 5 es significativamente menor y se acompañan con pérdida de la integridad vascular. Contra coloración nuclear con hematoxilina. Las barras indican $20 \mu \mathrm{m}$ 


\section{Discusión}

El presente estudio in vitro demuestra que el veneno entero de $B$. diporus induce a lesiones de desprendimiento de la membrana basal, causando separación de la dermis y epidermis, unión indispensable para mantener en posición normal la tercera falange dentro del casco. Pollitt y Daradka (1998) reportaron que la desintegración de la MB y la pérdida de su unión con las células basales de la epidermis parece ser uno de los primeros eventos patológicos que se producen en la laminitis aguda y podría ser el cambio que inicia el colapso de la arquitectura lamelar.

Paralelamente, en este estudio se verificó que el veneno causó lesiones importantes en los componentes de células epidérmicas basales. Este hallazgo es congruente con el trabajo de Teibler et al. (2017) con respecto al efecto del desprendimiento de células endoteliales de la membrana basal. Similares antecedentes fueron reportados por otros autores que estudiaron el efecto de metaloproteinasas de vipéridos, determinando que estos componentes de los venenos interfieren con las moléculas de adhesión entre células endoteliales y la matríz extracelular (Diaz et al., 2005; Tanjoni et al., 2005).

Las investigaciones evidencian que estas enzimas también están involucradas en la patogenia de la mionecrosis local (Gutiérrez et al., 1995), daño de piel (Rucavado et al., 2002), edema y otras reacciones asociadas con inflamación inducida por venenos (Gutiérrez et al., 1995; Moura da Silva et al., 1996). Así, las metaloproteinasas de veneno juegan un rol relevante en la patogenia del daño tisular local de dos maneras: a) por liberar el factor $\alpha$ de necrosis tumoral al activar al precursor unido a la membrana (Moura da Silva et al., 1996), facilitando la generación y liberación de una cascada de mediadores inflamatorios que contribuyen con el daño del tejido, y b) por inducir la síntesis de las MMP en tejidos afectados (Rucavado et al., 2002; Gutiérrez y Rucavado, 2000).
Los hallazgos de este estudio con respecto a la pérdida de la integridad causada por las MMP con disminución de la detección de L-5 son concordantes con lo reportado por Kaenulainen et al (1998), quienes indican que L-5 juega un rol esencial en la interacción con queratinocitos y que diferentes componentes del microambiente de la matriz extracelular interaccionan durante la cicatrización para restaurar la integridad de la membrana basal con L-5, independientemente del tipo de injuria experimental aplicada. Surge aquí una nueva perspectiva de desarrollo futuro, considerando que sería plausible pensar que, inhibiendo la acción de las MMP en los tejidos lamelares equinos, el tejido se reconstituiría con la sobreexpresión de L-5. Estudios adicionales deberán ser realizados para poder asegurar esta presunción.

Resulta interesante, por otra parte, tener en cuenta que L-5 se halla notablemente disminuida en los componentes estructurales de los vasos sanguíneos del pie equino por acción del veneno de $B$. diporus, conllevando a una hipoxia del microambiente lamelar. La hipoxia es un poderoso desencadenante de la apoptosis o muerte celular programada por la vía intrínseca, por lo que los desprendimientos de la unión dermo-epidermal con las alteraciones histológicas descritas en este estudio podrían estar reflejando tanto el efecto directo de las MMP del veneno, como el efecto indirecto de la baja tensión de oxígeno en la supervivencia de las células basales epidermales de las laminillas y estructuras vasculares. En este sentido y coincidentemente con lo observado, se reporta que los niveles de L-5 decrecen en células epiteliales de córneas cultivadas in vitro en condiciones hipoxicas y que estas sufren muerte celular por apoptosis frente a los cambios del potencial de membrana mitocondrial (Esco et al., 2001)

Estos resultados permiten inferir que las metaloproteinasas componentes de los venenos de $B$. diporus podrían repercutir en alteraciones de la vascularización y en la estructura de las láminas de cascos equinos en ac- 
cidentes ofídicos a través de la degradación de L-5. Este estudio constituye un aporte original a la comprensión de las bases subyacentes en la intoxicación ofídica por $B$. diporus y podría ser extrapolado a la clínica veterinaria para futuras intervenciones terapéuticas frente a este tipo de venenos.

\section{Literatura Citada}

1. Aberdam D, Aguzzi A, Baudoin C, Galliano MF, Ortonne JP, Meneguzzi G. 1994. Developmental expression of nicein adhesion protein (laminin-5) subunits suggests multiple morphogenic roles. Cell Adhes Commun 2: 115-29. doi: 10.3109/15419069409004431

2. Acosta O, Brem JJ. 1997. Secuelas de ofidismo en un equino de trabajo. Vet Arg 14(137): 474-478.

3. Acosta O, Teibler P, Leiva L, Ríos E, Negrette MS, Pollitt C. 2006. Equine laminitis: bites by Bothrops sp cause hoof lamellar pathology in the contralateral as well as in the bitten limb. Toxicon 48: 307-312. doi:10.1016/ j.toxicon.2006.06.010

4. Bailey SR, Adair HS, Reinemeyer CR, Morgan SJ, Brooks AC, Longhofer SL, Elliott J. 2009. Plasma concentrations of endotoxin and platelet activation in the developmental stage of oligofructose-induced laminitis. Vet Immunol Immunopathol 129: 167-173. doi: 10.1016/j.vetimm.2008.11.009

5. Bogado F, Nuñez S, Mussart NB, Leiva L, Acosta AC. 2013. Cambios clínicos, hemáticos y coagulativos consecuentes al aumento de anticuerpos en equinos productores de suero antiofídico. Rev Vet 24(1): 3-9. doi: 10.30972/ rv.v24i1.1141

6. Díaz C, Valverde L, Brenes O, Rucavado A, Gutierrez JM. 2005. Characterization of events associated with apoptosis/anoikis induced by snake venom metalloproteinase $\mathrm{BaP} 1$ on human endothelial cells. J Cell Biochem 94: 520528. doi: $10.1002 /$ jcb. 20322
7. Dickinson CE, Traut-Dargatz JL, Dragatz DA, Bennett DG, Knight AP. 1996. Rattlesnake venom poisoning in horses: 32 cases (1973-1993). J AmVet Med Assoc 208: 1866-1871.

8. Engvall E. 1993. Laminin variants: why, where and when? Kidney Int 43: 2-6. doi: 10.1038/ki.1993.2

9. Esco MA, Wang Z, McDermott ML, Kurpakus-Wheater M. 2001. Potential role for laminin 5 in hypoxia-mediated apoptosis of human corneal epithelial cells. J Cell Sci 114: 4033-4040.

10. García-Denegri ME. 2011. Aislamiento y caracterización de fosfolipasa $\left(\mathrm{PLA}_{2}\right)$ del veneno de Bothrops alternatus (víbora de la cruz) del nordeste argentino. Tesis Doctoral. Argentina: Univ. Nacional de Córdoba. 44 p.

11. Gay CC, Leiva LC, Maruñak S, Teibler P, Acosta de Pérez O. 2005. Proteolytic, edematogenic and myotoxic activities of a hemorrhagic metalloproteinase isolated from Bothrops alternatus venom. Toxicon 46: 546-554. doi: 10.1016/j.toxicon.2005.06.019

12. Gay CC, Maruñak SL, Teibler P, Ruiz $R$, Acosta de Pérez OC, Leiva LC. 2009. Systemic alterations induced by a Bothrops alternatus hemorrhagic metalloproteinase (baltergin) in mice. Toxicon 53: 53-59. doi: 10.1016/ j.toxicon.2008.10.010

13. Gutiérrez JM, Romero M, Nuñez J, Chaves F, Borkow G, Ovadia M. 1995. Skeletal muscle necrosis and regeneration after injection of $\mathrm{BaH} 1$, a hemorrhagic metalloproteinase isolated from the venom of the snake Bothrops asper (Terciopelo). Exp Mol Pathol 62: 28-41. doi: 10.1006/exmp.1995.1004

14. Gutiérrez JM, Rucavado A. 2000. Snake venom metalloproteinases: their role in the pathogenesis of the local tissue damage. Biochimie 82: 841-850. doi: 10.1016/S0300-9084(00)01163-9

15. Kaenulainen T, Häkkinen L, Hamidi S, Larjava T, Kallioinen M, Peltonen J, Salo T, et al. 1998. Laminin-5 expression is independent of the injury and the microenvironment during 
reepithelialization of wounds $\mathrm{J}$ Histochem Cytochem 46: 353-360. doi: 10.1177/002215549804600309

16. Katwa LC, Johnson PJ, Ganjam VK, Kreeger JM, Messer NT. 1999. Expression of endothelin in equine laminitis. Equine Vet J 31: 243-247. doi: 10.1111/j.2042-3306.1999.tb03180.x

17. LeBleu VS, Macdonald B, Kalluri $R$. 2007. Structure and function of basement membranes. Exp Biol Med (Maywood) 232: 1121-1129. doi: 10.3181/0703-MR-72

18. McGowan C. 2008. The role of insulin in endocrinopathic laminitis. J Eq Vet Sci 28: 603-607. doi: 10.1016/j.jevs.2008.08.004

19. Moore RM, Eades SC, Stokes AM. 2004. Evidence for vascular and enzymatic events in the pathophysiology of acute laminitis: which pathway is responsible for initiation of this process in horses? Equine Vet J 36: 204-209. doi: 10.2746/0425164044877116

20. Moura da Silva AM, Laing GD, Paine MJ, Dennison JM, Politi V, Crampton JM, Theakston RD. 1996. Processing of pro-tumor necrosis factor alpha by venom metalloproteinase: a hypothesis explaining local tissue damage following snake bite. Eur J Immunol 26: 2000-2005. doi: 10.1002/eji.1830260905

21. Oliveira AK, Paes AF, Asega $A F$, Camargo AC, Fox JW, Serrano SM. 2010. New insights into the structural elements involved in the skin haemorrhage induced by snake venom metalloproteinases. Thromb Haemost 104: 485497. doi: 10.1160/TH09-12-0855

22. Pestronk A, Parhad IM, Drachman DB, Price DL. 1982. Membrane myopathy: morphological similarities to Duchenne muscular dystrophy. Muscle Nerve 5: 209-214. doi: 10.1002/ mus. 880050306

23. Pollitt CC. 1996. Basement membrane pathology: a feature of acute equine laminitis. Equine Vet J 28: 38-46. doi: 10.1111/j.2042-3306.1996.tb01588.x
24. Pollitt CC. 1998a. The anatomy and physiology of the hoof wall. Equine Vet Educ 10: 318-325. doi: 10.1111/j.20423292.1998.tb00902.x

25. Pollitt CC. 1998b. El pie del caballo. Madrid, España: Ed. Harcourt-Brace. $208 \mathrm{p}$.

26. Pollitt CC, Daradka.1998. Equine laminitis basement membrane pathology: loss of type IV collagen, type VII collagen and laminin immune staining. Equine Vet J 26: 139-144. doi: 10.1111/ j.2042-3306.1998.tb05133.x

27. Ribeiro $R$, Mendes $H$, Valadares $R$, Paz C, Lage de Oliveira A, Spadeto O, Faleiro R. 2017. A novel equine hoof lamellar tissue biopsy technique. J Equine Vet Sci 49: 63-68. doi: 10.1016/ j.jevs.2016.10.007

28. Rousselle P, Lunstrum GP, Keene DR, Burgeson RE. 1991. Kalinin: an epithelium-specific basement membrane adhesion molecule that is a component of anchoring filaments. J Cell Biol 114: 567-576. doi: 10.1083/jcb.114.3.567

29. Rucavado A, Escalante T, Texeira CF, Fernandes CM, Diaz C, Gutierrez JM. 2002. Increments in cytokines and matrix metalloproteinases in skeletal muscle after injection of tissue-damaging toxins from the venom of the snake Botrops asper. Mediators Inflamm 11: 121-128. doi: 10.1080/09629350220131980

30. Tanjoni I, Weinlich R, Della-Casa MS, Clissa PB, Saldanha RF, de Freitas MS, Barja-Fidalgo C, et al. 2005. Jararhagin, a snake venom toxin, induces a specialized form of apoptosis (anoikis) selective to endothelial cells. Apoptosis 10: 851-861. doi: 0.1007/ s10495-005-2945-1

31. Teibler GP, Bustillo S, Dubiel CJ, Bogado EF, Maruñak SL. 2017. Daño vascular inducido en el pie equino por veneno de la serpiente Bothrops diporus. Rev Vet 28(1): 41-46. doi: 10.30972/ rv.v28i1.1297 
32. Texeira C, Cury Y, Moreira V, Picolo G, Chaves F. 2009. Inflammation induced by Bothrops asper venom. Toxicon 54: 67-76. doi: 10.1016/ j.toxicon.2009.03.019

33. Verrando P, Schofield O, IscidaYamamoto A, Aberdam D, Partouche O, Eady RAJ, Ortonne JP. 1993. Nicein (BM-600) in junctional epidermolysis bullosa: polyclonal antibodies provide new clues for pathogenic role. J Invest Dermatol 101: 738-743.

34. Vieira DF, Watanabe L, Santana CD, Marcussi S, Sampaio SV, Soares AM, Arni RK. 2004. Purification and characterization of jararassin-I, a thrombin-like enzyme from Bothrops jararaca snake venom. Acta Biochim Biophys Sin (Shanghai) 36: 798-802.

35. Visser MB, Pollitt CC. 2011. Immunohistochemical distribution of laminin-332 and collagen type IV in the basement membrane of normal horses and horses with induced laminitis. J Comp Pathol 145: 80-87. doi: 10.1016/ j.jcpa.2010.11.009

36. Wang WJ, Huang TF. 2002. Purification and characterization of a novel metalloproteinase, acurhagin, from Agkistrodon acutus venom. Thromb Haemost 87: 641-650.

37. Yurchenco PD, Schittny JC. 1990. Molecular architecture of basement membranes. FASEB J 4: 1577-1590. 